\title{
Lysosomotropic agents: impact on lysosomal membrane permeabilization and cell death
}

\author{
Ana M. Villamil Giraldo, Hanna Appelqvist, Thomas Ederth and Karin Öllinger
}

\section{Linköping University Post Print}

\section{Tweet}

N.B.: When citing this work, cite the original article.

Original Publication:

Ana M. Villamil Giraldo, Hanna Appelqvist, Thomas Ederth and Karin Öllinger, Lysosomotropic agents: impact on lysosomal membrane permeabilization and cell death, 2014, Biochemical Society Transactions, (42), 1460-1464.

http://dx.doi.org/10.1042/BST20140145

Copyright: Portland Press

http://www.portlandpress.com/

Postprint available at: Linköping University Electronic Press

http://urn.kb.se/resolve?urn=urn:nbn:se:liu:diva-111745 


\title{
Lysosomotropic agents - impact on lysosomal membrane permeabilization and cell death
}

\author{
Ana M Villamil Giraldo ${ }^{1}$, Hanna Appelqvist ${ }^{2}$, Thomas Ederth ${ }^{3}$, Karin Öllinger ${ }^{1 *}$ \\ ${ }^{1}$ Division of Cell Biology, Department of Clinical and Experimental Medicine, Faculty of \\ Health Sciences, Linköping University, Pathology Clinic, County Council of Östergötland, \\ Linköping, Sweden. \\ ${ }^{2}$ Division of Chemistry, ${ }^{3}$ Division of Molecular Physics, Department of Physics, Chemistry \\ and Biology, Linköping University, Linköping, Sweden
}

To whom correspondence should be addressed.

karin.ollinger@liu.se

Keywords: Lysosome, lysosomal membrane, permeabilization, sphingosine, MSDH, detergent 


\begin{abstract}
Lysosomes are acidic organelles essential for degradation, signaling and cell homeostasis. In addition, they play a key role in cell death. Permeabilization of the lysosomal membrane and release of hydrolytic enzymes to the cytosol is accompanying apoptosis signaling in several systems. The regulatory mechanism of lysosomal stability is however poorly understood. Lipophilic or amphiphilic compounds with a basic moiety will become protonated and trapped within lysosomes and such lysosomotropic behavior is also found in many pharmacological drugs. The natural sphingolipid sphingosine exhibits lysosomotropic detergent ability and is an endogenous candidate for controlling lysosomal membrane permeabilization. The lysosomotropic properties of certain detergents might in the future be of use in lysosome-targeting anti-cancer drugs and drug delivery system. This review summarizes the current knowledge on the targeting and permeabilizing properties of lysosomotropic detergents from a cellular and physico-chemical perspective.
\end{abstract}




\section{The lysosome}

Lysosomes were originally described in the 1950s by Christian de Duve and are the key degradative compartment of the cell. They are advanced organelles involved in many cellular processes and are considered crucial regulators of cell homeostasis [reviewed in 1]. The interior is acidic with a $\mathrm{pH}<5$ and contains over 50 hydrolases, able to degrade all constituents of the cell. Lysosomes are limited by a single 7-10 nm phospholipid-bilayer that is functioning as an interface to regulate communication between the lysosomal lumen and the cytosol and therefore transversed by several membrane proteins [2]. In addition to the limiting lysosomal membrane, lysosomes and late endosomes contain intralysosomal membranes, which represent the main site of degradation within this organelle [3]. Lysosomal membrane proteins are generally heavily glycosylated at their luminal domain and form a glycocalyx, which is suggested to protect the membrane from the action of the hydrolytic interior [4]. The lysosomal membrane also facilitates interaction and fusion with other cellular compartments, including endosomes, autophagosomes and the plasma membrane [5].

\section{Lysosomal membrane permeabilization in cell death}

Due to their high hydrolase content, leakage of lysosomal content to the cytosol is potentially harmful to the cell. Partial permeabilization of the membrane induces apoptosis while massive lysosomal rupture induces necrosis [6,7]. Lysosomal membrane permeabilization (LMP) and release of the lysosomal proteases, the cathepsins, to the cytosol has been shown to be the critical step in lysosomal mediated apoptosis [8-12]. Although the proteolytic activity of the cathepsins is dependent on acidic $\mathrm{pH}$, their mechanism of action in the cytosol has also been found to be by proteolytic processing of cytosolic substrates. This might be explained by stabilization of the cathepsins by substrate binding or cytosolic acidification by protons released from the lysosome [13-15]. Bid processing into its pro-apoptotic truncated form (tBid) is the most studied cathepsin substrate [11,16-17]. Other substrates have also been identified and has been reviewed elsewhere [1]. Following LMP the downstream signaling often involves activation of the caspase cascade, via the intrinsic $[18,19]$ and extrinsic [20] pathways. The mechanism of LMP by which lysosomal content escapes the lysosomes remains elusive, although several compounds have been identified as both stimulating and preventing the loss of lysosomal integrity [reviewed in 19]. The possibility of inducing cell death through LMP appears interesting in tumor treatment because the apoptosis mechanism is frequently impaired in cancer cells, while lysosomes could be considered an Achilles' heel.

\section{Lysosomotropism}

The acidic interior of lysosomes makes them susceptible to the accumulation of weak bases able to diffuse across the lysosomal membrane. As they reach this compartment these substances become protonated and, as charged molecules, their diffusion back into the cytosol is severely hindered [21]. Such substances are referred to as lysosomotropic agents, and via this mechanism, trapped substances may accumulate to concentrations hundredfold that of the cytosolic concentration [22] (Figure 1). Several well-known drugs used in the clinic have documented lysosomotropic abilities including the antimalarial drug chloroquine, several antipsychotic drugs (chlorpromazine, thioridazine, and aripiprazole) and antidepressants (desipramine, imipramine, and clomipramine). A recent screening of lysosomotropism, [23] found that drugs with a $C \log \mathrm{P}>2$ (partition coefficient of the neutral species of a compound between octanol and water) and pKa between 6.5 and 11, caused lysosomal accumulation. The lysosomotropism in itself could probably contribute to cytotoxicity [24] and several of these compounds have been shown to cause LMP. The mechanistic explanation to this differs 
depending on their chemical structure. Lysosomal lysis due to accumulation of the nonpermeable charged substances such as free amino acids after metabolism of L-Leucyl-Lleucine methyl ester (LeuLeuOMe) or Gly-Phe- $\beta$-naphtylamide could build up an osmotic pressure across the lysosomal membrane, which results in the inflow of water [12,25]. Other toxic effects of lysosomotropic drugs are attributed to inhibition of acid sphingomyelinase (ASM), a lysosomal enzyme that catalyzes the degradation of sphingomyelin to ceramide [26]. In most cases, however, a mechanistic explanation to LMP is lacking.

\section{Lysosomotropic detergents}

An interesting group of substances are the lysosomotropic detergents (LDs), which according to their amphiphilic nature, can partition in the phospholipid bilayer and translocate across membranes as uncharged molecules. Lysosomotropic amines contain a moderately basic amino group with a pKa of 5-9 and are able to passively diffuse across cell membranes. If the compound also contains a long hydrophobic chain, it will act by burying its hydrophobic tail in the membrane with the hydrophilic protonated head group facing the aqueous interior of the lysosomes [27]. The amine will accumulate inside the lysosomes until the concentration is sufficient to solubilize the lysosomal membrane [28]. In contrast to other detergents that kill cells by acting at the plasma membrane, lysosomotropic detergents primarily act from within the lysosomes. The lines of evidence leading to this conclusion include the absence of cytotoxicity of LDs towards cells lacking lysosomes [e.g. erythrocytes], the dependence of cytotoxicity on the capacity of LDs of being protonated and on their hydrocarbon chain length, the intracellular vacuolization preceding cell destruction and the protection against cytotoxicity exerted by raising lysosomal $\mathrm{pH}[27,29,30]$.

Different LDs have been extensively studied using cell models and can be used to study the cellular effect of targeted lysosomal permeabilization. O-methyl-serine dodecylamine hydrochloride (MSDH), an amine with a 12-carbon hydrophobic chain and a pKa of 5.9 [28] induces apoptotic cell death in several cellular systems including macrophage-like cells, and human fibroblasts $[6,17]$. The apoptosis-inducing mechanism relies on the activity of lysosomal proteases released to the cytosol followed by release of cytochrome $\mathrm{c}$ from mitochondria and initiation of the caspase cascade. Noteworthy, MSDH-induced lysosomal leakage is detected without complete disintegration or lysis of the organelle.

Immunofluorescence staining of LAMP-2 (lysosomal associated membrane protein-2) shows that the lysosomal spherical shape is clearly visible even after considerable amounts of proteinases are found in the cytosol [31]. The LDs N-dodecylimidazole also shows detergent properties and the cell killing mechanism was dependent on acidic conditions and functioning lysosomes [29]. The dependence of lysosomal enzymes for cell killing was also elegantly shown by the use of fibroblasts from patients with mucolipidosis II (I-cell disease), which lack the ability to add mannose-6-phosphate to pro-cathepsins and target them to lysosomes. Thus, $\mathrm{N}$-dodecylimidazole toxicity was reduced in I-cell fibroblasts as compared to normal fibroblasts [30]. The amphiphilic amine seramisine, a $\sigma-2$ receptor ligand and potent anticancer agent, has been found to cause rise in the lysosomal $\mathrm{pH}$ and cause cell death in tumor cells in a detergent-like manner. After treatment the lysosomes were significantly more prone to disintegrate in isotonic conditions in vitro than lysosomes from untreated cells. This effect was further emphasized when the isolated lysosomes were exposed to hypotonic stress [32]. A 
recent study was, however unable to verify the lysosomotropic effect of seramisine in HaCaT cells [33].

\section{Sphingosine - the endogenous LD?}

Sphingosine is a bioactive lipid of cellular membranes that is involved in numerous physiological functions. It belongs to the sphingolipids and is implicated in LMP and apoptosis $[7,34,35]$. Sphingosine is proposed to accumulate inside lysosomes and to permeabilize the membrane in a detergent-like fashion. Sphingosine is generated in the lysosome by the sequential action of aSMase and acid ceramidase, which convert sphingomyelin into ceramide and, subsequently, sphingosine (Figure 2). In contrast to sphingosine, sphingomyelin has been shown to protect against lysosomal destabilization [16]. The addition of sphingosine to isolated lysosomes induces membrane destabilization, which is dependent on the presence of cathepsin B [35]. In apoptotic cells, the levels of sphingosine may increase due to the enhanced activity of aSMase and cathepsin B-mediated degradation of sphingosine kinase-1 [36], which normally converts pro-apoptotic sphingosine into antiapoptotic sphingosine-1-phosphate. This effect may explain, at least in part, the dependence of sphingosine on cathepsin B for efficient LMP-induction. On the other hand, it was demonstrated that apoptosis could be induced by sphingosine acting as a typical lysosomotropic detergent [7], i.e. by being accumulated in the lysosomal acidic compartment and selectively permeabilizing the lysosomal membrane. Sphingosine was shown to produce early lysosomal leakage as assessed by the relocation of fluorescent dyes and lysosomal enzymes and this lysosomal membrane disruption was shown to occur before any sign of apoptosis could be detected. The authors also showed that raising lysosomal $\mathrm{pH}$ protected the cells from sphingosine-induced apoptosis, which points to the importance of $\mathrm{pH}$-dependent accumulation of sphingosine inside lysosomes.

Addition of exogenous sphingosine was shown to induce cell death $[37,38]$ with concomitant alteration of components of the apoptotic signaling pathway. However, the interconversion of sphingosine into ceramide and the difficulties in inhibiting this metabolic step $[39,40]$ complicates a final conclusion about the effect of sphingosine on classic apoptotic cascades independent of that well established for ceramide [41].

\section{Model membrane permeabilization by sphingosine}

As mentioned above numerous cell studies have been carried out using several different LDs. On the contrary, the physico-chemical mechanism of permeabilization using model membranes has only been examined for the natural LD sphingosine. Its chemical structure consists of a long hydrocarbon chain, usually C18 and C20, which contains an amine group that can become protonated being its $\mathrm{pKa} \sim 6.6[42,43]$. Its apparent critical aggregation concentration, i.e. the concentration above which sphingosine monomers start forming aggregates, depends upon $\mathrm{pH}$ [42] and it was found to be $\sim 2.4$-fold higher for the protonated form. The structure of sphingosine aggregates is also dependent upon $\mathrm{pH}$. Increasing uncharged sphingosine concentration leads to an unrestrained growth in aggregates size while increasing protonated sphingosine concentration leads to an increase in aggregates concentration. These results were explained in terms of a shift in the hydrogen-bonding 
network from intra to intermolecular when sphingosine becomes protonated. The change in aggregate structure can also be understood in terms of packing parameters, where the increased headgroup repulsion between charged sphingosines changes the effective headgroup area in the aggregate, inducing a greater curvature on the aggregate surface. Sphingosine partitions in and stabilizes cholesterol-sphingomyelin ordered domains [44] and it also rigidifies membranes composed of phosphatidylcholine [PC], phosphatidylserine, phosphatidic acid or their mixtures [45]. The ordering effect of sphingosine on fluid PC membranes or on PC/sphingomyelin/cholesterol raft-mimicking membranes was shown to depend on sphingosine concentration and on $\mathrm{pH}$. Gel domains enriched in sphingosine were more easily formed at neutral $\mathrm{pH}$ where most of sphingosine molecules are uncharged [46]. Model membranes were shown to be permeabilized by sphingosine [47] and the extent of permeabilization was shown to depend on the lipid composition. Interestingly, the permeabilization exerted by sphingosine was not a consequence of a detergent-like effect, i.e. membranes were not solubilized by sphingosine, or by the transient formation of nonlamellar inverted structures. Rather, the increased permeability induced by sphingosine was a consequence of its selective partition and resulting rigidification of less fluid lipid domains, which creates defects at the interfaces between these domains and coexisting more fluid ones. Sphingosine was also shown to form channels in biological membranes of different lipidic composition such as mitochondria and plasma membrane [39]. These channels were characterized as having short open lifetimes and diameters not large enough $(\sim 2 \mathrm{~nm})$ to allow for the leakage of apoptotic proteins from mitochondria. The above mentioned physicochemical studies point to the importance of the effect of $\mathrm{pH}$ on the permeabilization properties of LDs.

\section{Future challenges}

The ability to target the lysosomal compartment and specifically permeabilize the lysosomal membrane was early identified as potentially useful to trigger cell death. In this sense LDs appear as particular interesting molecules because they not only share this ability with lysosomotropic agents in general but also, being amphiphilic molecules, spontaneously organize in aggregates that could serve as carrier systems. In order to exploit the targeting and permeabilizing properties of LDs a detailed understanding of their physicochemical behavior is required. Thus, it becomes necessary to carry out studies using model membranes. These studies will in turn provide complementary information on the physiological regulation of LMP. 


\section{References}

1. Appelqvist, H., Wäster, P., Kågedal, K and Öllinger, K. (2013) The lysosome: from waste bag to potential therapeutic target. J Mol Cell Biol. 5, 214-26

2. Saftig, P., Schröder, B and Blanz, J. (2010). Lysosomal membrane proteins: life between acid and neutral conditions. Biochem. Soc. Trans. 38, 1420-1423

3. Schulze, H., Kolter, T and Sandhoff, K. (2009). Principles of lysosomal membrane degradation: Cellular topology and biochemistry of lysosomal lipid degradation. Biochim. Biophys. Acta 1793, 674-683

4. Granger, B.L., Green, S.A., Gabel, C.A., Howe, C.L., Mellman, I. and Helenius, A. (1990). Characterization and cloning of lgp110, a lysosomal membrane glycoprotein from mouse and rat cells. J. Biol. Chem. 265, 12036-12043

5. Schröder, B.A., Wrocklage, C., Hasilik, A and Saftig, P. (2010) The proteome of lysosomes. Proteomics. 10, 4053-76

6. Li, W., Yuan, X., Nordgren, G., Dalen, H., Dubowchik, G.M., Firestone, R.A. and Brunk, U.T. (2000) Induction of cell death by the lysosomotropic detergent MSDH. FEBS Lett. 470, 35-9

7. Kågedal, K., Zhao, M., Svensson, I. and Brunk, U.T. (2001) Sphingosine-induced apoptosis is dependent on lysosomal proteases. Biochem J. 359, 335-43

8. Roberg, K. and Öllinger, K. (1998). Oxidative stress causes relocation of the lysosomal enzyme cathepsin $\mathrm{D}$ with ensuing apoptosis in neonatal rat cardiomyocytes. Am. J. Pathol. 152, 1151-1156

9. Stoka, V., Turk, B., Schendel, S.L., Kim, T.H., Cirman, T., Snipas, S.J., Ellerby, L.M., Bredesen, D., Freeze, H., Abrahamson, M., Bromme, D., Krajewski, S., Reed, J.C., Yin, X.M., Turk, V. and Salvesen, G.S. (2001) Lysosomal protease pathways to apoptosis. Cleavage of bid, not pro-caspases, is the most likely route. J Biol Chem. 276, 3149-57

10. Roberg, K., Kågedal, K. and Öllinger, K. (2002). Microinjection of cathepsin D induces caspase-dependent apoptosis in fibroblasts. Am. J. Pathol. 161, 89-96

11. Cirman, T., Oresic, K., Mazovec, G.D., Turk V., Reed J.C. and Myers R.M. (2004). Selective disruption of lysosomes in HeLa cells triggers apoptosis mediated by cleavage of Bid by multiple papain-like lysosomal cathepsins. J. Biol. Chem. 279, 3578-3587

12. Uchimoto, T., Nohara, H., Kamehara, R., Iwamura, M., Watanabe, N. and Kobayashi, Y. (1999) Mechanism of apoptosis induced by a lysosomotropic agent, L-Leucyl-LLeucine methyl ester. Apoptosis. 4, 357-62

13. Kirschke, H., Wiederanders, B., Bromme, D. and Rinne, A. (1989). Cathepsin S from bovine spleen. Purification, distribution, intracellular localization and action on proteins. Biochem. J. 264, 467-473

14. Gottlieb, R.A., Giesing, H.A., Zhu, J.Y., Engler R.L. and Babior B.M. (1995). Cell acidification in apoptosis: granulocyte colony-stimulating factor delays programmed cell death in neutrophils by up-regulating the vacuolar H(+)-ATPase. Proc. Natl. Acad. Sci. U.S.A. 92, 5965-5968

15. Nilsson, C., Johansson, U., Johansson, A.C., Kådegal K. and Öllinger K. (2006). Cytosolic acidification and lysosomal alkalinization during TNF-alpha induced apoptosis in U937 cells. Apoptosis 11, 1149-1159

16. Caruso, J.A., Mathieu, P.A. and Reiners, J.J. Jr. (2005). Sphingomyelins suppress the targeted disruption of lysosomes/endosomes by the photosensitizer NPe6 during photodynamic therapy. Biochem. J. 392, 325-334 
17. Appelqvist, H., Johansson, A.C., Linderoth, E., Johansson, U., Antonsson, B., Steinfeld, R., Kådegal, K. and Öllinger, K. (2012). Lysosome-mediated apoptosis is associated with cathepsin D-specific processing of Bid at Phe24, Trp48, and Phe183. Ann. Clin. Lab. Sci. 42, 231-242

18. Boya, P., Andreau, K., Poncet, D., Zamzami, N., Perfettini, J.L., Metivier, D., Ojcius, D.M., Jäättelä, M. and Kroemer, G. (2003) Lysosomal membrane permeabilization induces cell death in a mitochondrion-dependent fashion. J Exp Med. 197, 1323-34.

19. Johansson, A.C., Appelqvist, H., Nilsson, C., Kådegal K., Roberg K. and Öllinger K. (2010). Regulation of apoptosis-associated lysosomal membrane permeabilization. Apoptosis 15, 527-540

20. Guicciardi, M.E., Deussing, J., Miyoshi, H., Bronk, S.F., Svingen P.A., Peters, C., Kaufmann, S.H. and Gores, G.J. (2000). Cathepsin B contributes to TNF-alphamediated hepatocyte apoptosis by promoting mitochondrial release of cytochrome c. J. Clin. Invest. 106, 1127-1137

21. de Duve, C., de Barsy, T., Poole, B., Trouet, A., Tulkens, P. and Van Hoof , F. (1974) Commentary. Lysosomotropic agents. Biochem Pharmacol. 23, 2495-531

22. Oda, K., Koriyama, Y., Yamada, E. and Ikehara, Y. (1986) Effects of weakly basic amines on proteolytic processing and terminal glycosylation of secretory proteins in cultured rat hepatocytes. Biochem J. 240, 739-45

23. Nadanaciva, S., Lu, S., Gebhard, D.F., Jessen, B.A., Pennie, W.D. and Will, Y. (2011) A high content screening assay for identifying lysosomotropic compounds. Toxicol In Vitro. 25, 715-23

24. Walls, K.C., Ghosh, A.P., Franklin, A.V., Klocke, B.J., Ballestas, M., Shacka, J.J., Zhang, J. and Roth, K.A. (2010) Lysosome dysfunction triggers Atg7-dependent neural apoptosis. J. Biol. Chem. 285, 10497-10507

25. Thiele, D.L. and Lipsky, P.E. (1990) Mechanism of L-leucyl-L-leucine methyl estermediated killing of cytotoxic lymphocytes: dependence on a lysosomal thiol protease, dipeptidyl peptidase I, that is enriched in these cells. Proc Natl Acad Sci USA. 87,83-7

26. Kornhuber, J., Tripal, P., Reichel, M., Terfloth, L., Bleich, S., Wiltfang, J. and Gulbins E. (2008) Identification of new functional inhibitors of acid sphingomyelinase using a structure-property-activity relation model J. Med. Chem. 51, 219-237

27. Firestone, R.A., Pisano, J.M. and Bonney, R.J. (1979) Lysosomotropic agents. 1. Synthesis and cytotoxic action of lysosomotropic detergents. J Med Chem. 22, 1130-3

28. Dubowchik, G.M., Gawlak, S.L. and Firestone, R.A. (1995) The in vitro effects of three lysosomotropic detergents against three human tumor cell lines Bioorg. Med. Chem. Lett. 5, 893-898

29. Miller, D.K., Griffiths, E., Lenard, J. and Firestone, R.A. (1983) Cell killing by lysosomotropic detergents. J Cell Biol. 97, 1841-51

30. Wilson, P.D., Firestone, R.A. and Lenard, J. (1987) The role of lysosomal enzymes in killing of mammalian cells by the lysosomotropic detergent $\mathrm{N}$-dodecylimidazole. J Cell Biol. 104, 1223-9

31. Appelqvist, H., Nilsson, C., Garner, B., Brown, A.J., Kågedal, K. and Ollinger, K. (2011) Attenuation of the lysosomal death pathway by lysosomal cholesterol accumulation. Am J Pathol. 178, 629-39

32. Ostenfeld, M.S., Høyer-Hansen, M., Bastholm, L., Fehrenbacher, N., Olsen, O.D., Groth-Pedersen, L., Puustinen, P., Kirkegaard-Sørensen, T., Nylandsted, J., Farkas, T. and Jäättelä, M. (2008) Anti-cancer agent siramesine is a lysosomotropic detergent that induces cytoprotective autophagosome accumulation. Autophagy. 4, 487-99

33. Česen, M.H., Repnik, U., Turk, V. and Turk, B. (2013) Siramesine triggers cell death through destabilisation of mitochondria, but not lysosomes. Cell Death Dis 4,e818 
34. Ullio, C., Casas, J., Brunk, U.T., Sala, G., Fabriàs, G., Ghidoni, R., Bonelli, G., Baccino, F.M. and Autelli, R. (2012) Sphingosine mediates TNF $\alpha$-induced lysosomal membrane permeabilization and ensuing programmed cell death in hepatoma cells. $\mathbf{J}$ Lipid Res. 53, 1134-43

35. Werneburg, N.W., Guicciardi, M.E., Bronk, S.F. Gores, G.J. (2002). Tumor necrosis factor-alpha-associated lysosomal permeabilization is cathepsin B dependent. Am. J. Physiol. Gastrointest. Liver. Physiol. 283, G947-956

36. Taha, T.A., Kitatani, K., Bielawski, J., Cho, W., Hannun, Y.A. and Obeid, L.M. (2005) Tumor necrosis factor induces the loss of sphingosine kinase-1 by a cathepsin B-dependent mechanism J Biol Chem. 280, 17196-202

37. Ohta, H., Yatomi, Y., Sweeney, E.A., Hakomori, S. and Igarashi, Y. (1994) A possible role of sphingosine in induction of apoptosis by tumor necrosis factor-alpha in human neutrophils. FEBS Lett. 355, 267-70

38. Cuvillier, O., Nava, V.E., Murthy, S.K., Edsall, L.C., Levade, T., Milstien, S. and Spiegel, S. (2001) Sphingosine generation, cytochrome c release, and activation of caspase-7 in doxorubicin-induced apoptosis of MCF7 breast adenocarcinoma cells. Cell Death Differ. 8, 162-71

39. Siskind, L.J., Fluss, S., Bui, M. and Colombini, M. (2005) Sphingosine forms channels in membranes that differ greatly from those formed by ceramide. J Bioenerg Biomembr. 37, 227-36

40. Bionda, C., Portoukalian, J., Schmitt, D., Rodriguez-Lafrasse, C. and Ardail, D. (2004) Subcellular compartmentalization of ceramide metabolism: MAM (mitochondria-associated membrane) and/or mitochondria? Biochem J. 382, 527-33

41. Morad, S.A. and Cabot, M.C. (2013) Ceramide-orchestrated signalling in cancer cells. Nat Rev Cancer. 13:51-65

42. Sasaki, H., Arai, H., Cocco, M.J. and White, S.H. (2009) pH dependence of sphingosine aggregation. Biophys J. 96, 2727-33

43. Merrill, A.H. Jr, Nimkar, S., Menaldino, D., Hannun, Y.A., Loomis, C., Bell, R.M., Tyagi, S.R., Lambeth, J.D., Stevens, V.L. and Hunter, R. (1989) Structural requirements for long-chain (sphingoid) base inhibition of protein kinase $\mathrm{C}$ in vitro and for the cellular effects of these compounds. Biochemistry. 28, 3138-45

44. Alanko, S.M., Halling, K.K., Maunula, S., Slotte, J.P. and Ramstedt, B. (2005) Displacement of sterols from sterol/sphingomyelin domains in fluid bilayer membranes by competing molecules. Biochim Biophys Acta. 1715, 111-21

45. Kõiv, A., Mustonen, P. and Kinnunen, P.K. (1993) Influence of sphingosine on the thermal phase behaviour of neutral and acidic phospholipid liposomes. Chem Phys Lipids. 66, 123-34

46. Zupancic, E., Carreira, A.C., de Almeida, R.F. and Silva, L.C. (2014) Biophysical Implications of Sphingosine Accumulation in Membrane Properties at Neutral and Acidic pH. J Phys Chem B. 118, 4858-66

47. Contreras, F.X., Sot, J., Alonso, A. and Goñi, F.M. (2006) Sphingosine increases the permeability of model and cell membranes. Biophys J. 90, 4085-92 


\section{Figures}

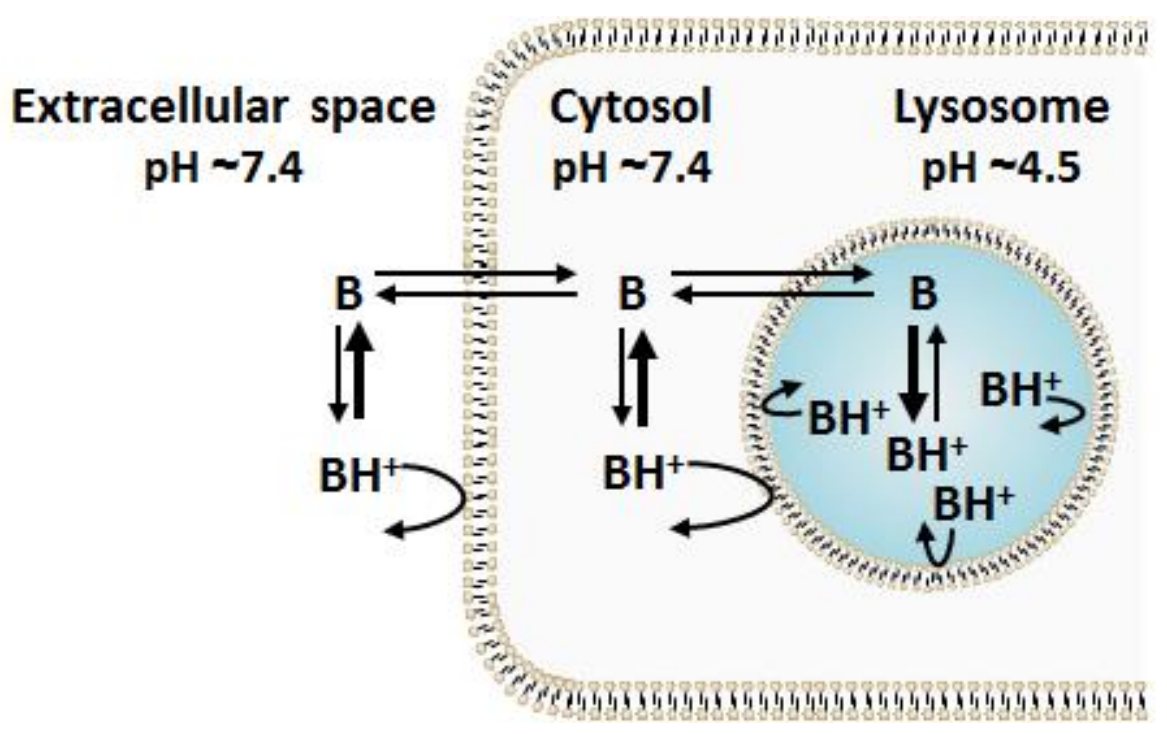

Figure 1. A lysosomotropic agent accumulates in lysosomes due to ion trapping. A weakly basic amine (B) will, in its unprotonated form, passively diffuse through cell membranes. In the acidic interior of lysosomes, the amine will become charged and can no longer pass through the lysosomal membrane, thus accumulating in the lysosomal compartment. 


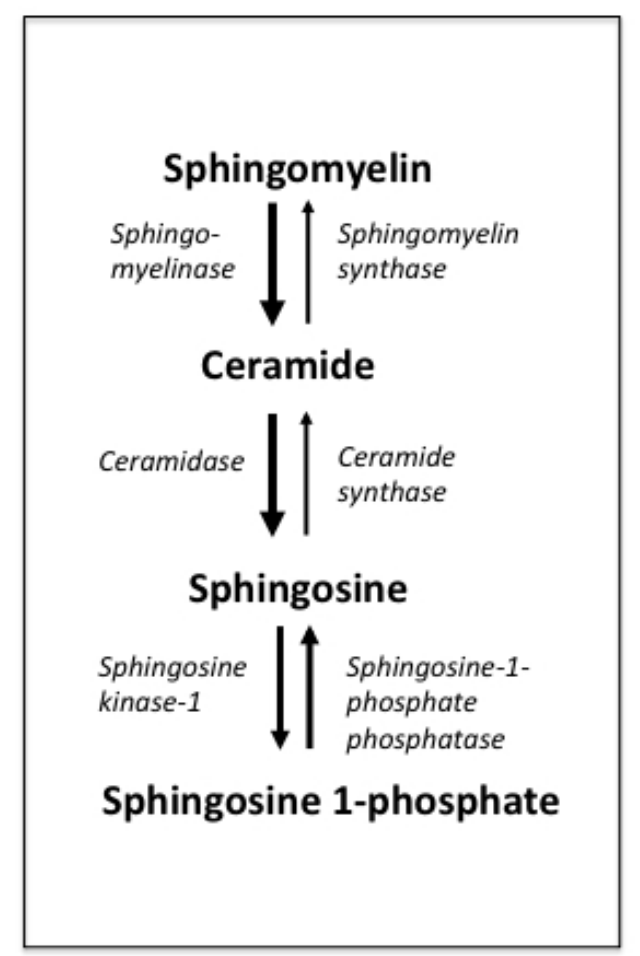

Figure 2. Conversion of sphingomyelin into sphingosine. Sphingomyelin is converted into ceramide, a reaction catalyzed by sphingomyelinase. By the action of ceramidase, ceramide is transformed into sphingosine. Sphingosine can accumulate inside lysosomes and induce lysosomal membrane permeabilization in a detergent-like fashion. Sphingosine can also be converted to sphingosine-1-phosphate, which is considered anti-apoptotic. 